\title{
A patient with plasmablastic lymphoma achieving long-term complete remission after thalidomide-dexamethasone induction and double autologous stem cell transplantation: a case report
}

\author{
Alessandro Broccoli', Laura Nanni, Vittorio Stefoni, Claudio Agostinelli, Lisa Argnani, Michele Cavo \\ and Pier Luigi Zinzani
}

\begin{abstract}
Background: No standard of care is established for plasmablastic lymphoma (PBL) and prognosis remains extremely poor, given that patients relapse early after chemotherapy and display resistance to commonly applied cytostatic drugs.

Case presentation: We report a case of nodal, HIV-unrelated PBL in a patient who achieved and maintained a very long lasting complete remission after an intensive therapy consisting consisting of thalidomide plus dexamethasone followed by a consolidation with double autologous stem cell transplantation. Our approach was based on the full application of a standard multiple myeloma treatment and, to the best of our knowledge, it represents the only reported experience so far. This treatment was overall well tolerated.
\end{abstract}

Conclusions: Multiple myeloma-like treatment may represent a possible alternative to intensive lymphoma-directed therapies.

\section{Background}

Plasmablastic lymphoma (PBL) is a rare and highly aggressive subtype of diffuse large B-cell lymphoma, characterized by diffuse proliferation of large neoplastic cells which resemble B-cell immunoblasts but have a plasma cell immunophenotype [1]. No standard of care is established for this disease and prognosis remains extremely poor, given that patients relapse early after chemotherapy and display resistance to commonly applied cytostatic drugs. We herein report a case of nodal, HIV-unrelated PBL in a patient who achieved and maintained a very long lasting complete remission after an intensive multiple myeloma (MM)-like treatment.

* Correspondence: alessandro.broccoli@studio.unibo.it Institute of Haematology "L. e A. Seràgnoli", University of Bologna, Via Massarenti, 9 -40138 Bologna, Italy

\section{Case presentation}

A 46-year old Italian female presented in June 2007 with 3 enlarged lymph nodes in her left groin without any systemic symptoms. She had no significant comorbidities and no underlying immunosuppression. An excisional biopsy showed diffuse proliferation of large lymphoid cells with several interspersed macrophages, which imparted a "starry-sky" appearance to the infiltrate (Fig. 1). Immunohistochemical examination revealed that tumor cells were negative for CD20, CD79a, CD5, CD30, PAX5, Bcl-2 and Bcl-6, while showing positivity for CD138, MUM1 and $\lambda$ light chain restriction (Fig. 2). Anaplastic large-cell lymphoma kinase (ALK) and human herpes virus 8 (HHV-8) were also tested by immunohistochemistry, and were both negative. The Ki-67 proliferation index was 98\%. Epstein-Barr virus (EBV)-encoded RNA in situ hybridization (EBER) was negative. A diagnosis of PBL 


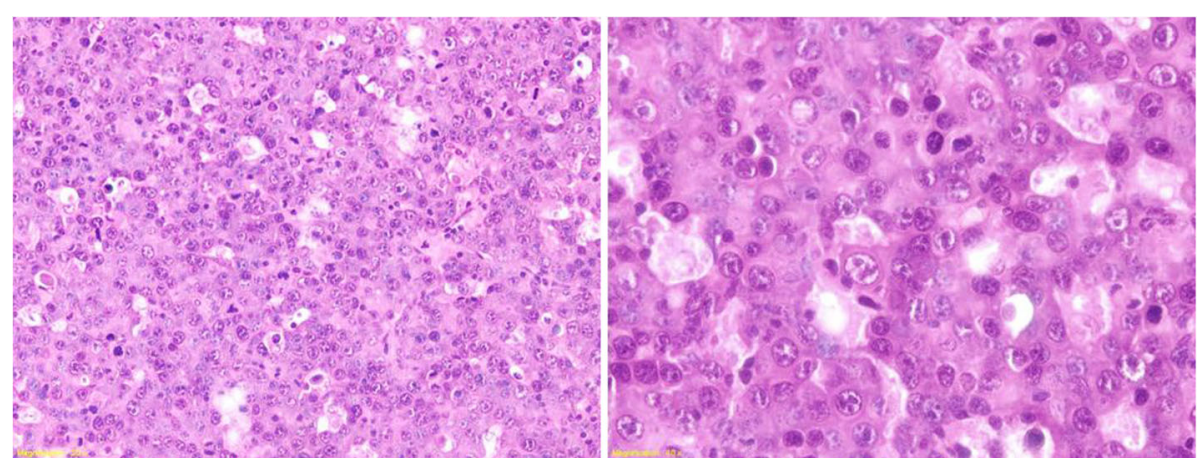

Fig. 1 Hematoxylin-eosin staining (20x and 40x magnification) showing macrophages admixed to plasmoblasts conferring a "starry-sky" appearance

was made. A total body computed tomography (CT) scan showed at least five adenopathies in her left groin, the largest of which was $22 \times 17 \mathrm{~mm}$. A positron emission tomography (PET) scan confirmed several hypermetabolic lymph nodes in both groins and pelvis (SUVmax 11-14). Biopsy of the iliac crest showed no marrow involvement.

On admission, laboratory data were the following: white blood cells $9500 / \mu \mathrm{L}$, hemoglobin $12.5 \mathrm{~g} / \mathrm{dL}$, platelets $313,000 / \mu \mathrm{L}$; lactate dehydrogenase, calcium, serum proteins and immunoglobulin levels were all within normal ranges; protein electrophoresis and immunofixation showed no monoclonal component. Serology for human immunodeficiency virus (HIV) was negative.

From July to September 2007, the patient received 3 cycles of thalidomide and dexamethasone: thalidomide was given at the dose of $100 \mathrm{mg} /$ day and dexamethasone at the dose of $40 \mathrm{mg} /$ day on days $1-4$ and 9-12 of each cycle. The rationale for the use of thalidomide in PBL relied upon the notion that increased angiogenesis plays a major role in aggressive lymphomas, as well as in MM [2]. The post-induction PET scan showed no metabolically active lesions, thus being compatible with a complete response (CR).
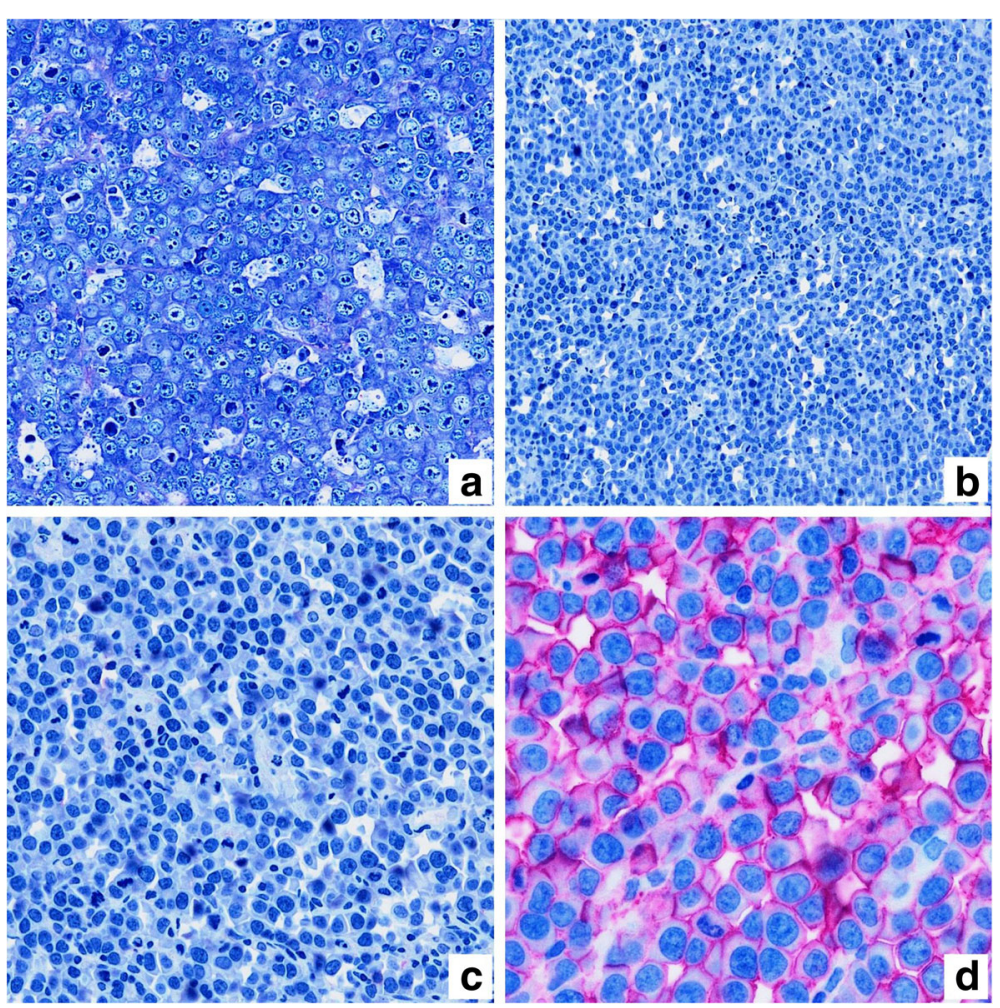

Fig. 2 Panel a: Giemsa stain (20x magnification). Neoplastic elements are negative for CD20 (panel b, 20x magnification) and CD30 (panel c, 40x magnification), whereas they stain positively for the plasma cell marker CD138 (panel d, 40x magnification) 
Afterwards, the patient received maintenance therapy with thalidomide for 2 months, followed by peripheral blood stem cell (PBSC) mobilization achieved by the administration of high dose cyclophosphamide $\left(7 \mathrm{~g} / \mathrm{m}^{2}\right)$ and granulocyte-colony stimulating factor (G-CSF): $7.2 \times 10^{6} \mathrm{CD}^{2} 4^{+} / \mathrm{kg}$ were harvested after a single apheresis. Following this procedure, the patient went back on thalidomide-dexamethasone for 3 more months, then she underwent an autologous stem cell transplantation (ASCT), conditioned with melphalan, $200 \mathrm{mg} / \mathrm{m}^{2}$. $2.5 \times 10^{6} \mathrm{CD}^{2} 4^{+} / \mathrm{kg}$ PBSC were reinfused. The PET scan performed one month after ASCT confirmed a CR. In light of the good response achieved so far, it was decided to proceed with a second ASCT. The patient again received thalidomide-dexamethasone for 3 months, then $2.5 \times 10^{6} \mathrm{CD}^{+} 4^{+} / \mathrm{kg}$ PBSC were reinfused 2 days after melphalan conditioning. A CR was clinically and metabolically confirmed by PET scan after the second ASCT. The patient is now healthy and in durable $\mathrm{CR}$ after at least 10 years.

This retrospective study was approved by our institutional board and by our Ethical Committee (Comitato Etico Indipendente Policlinico S.Orsola-Malpighi di Bologna) and has been performed in accordance with the ethical standards of the Declaration of Helsinki. Patient provided a written informed consent to publish her data.

\section{Discussion and conclusions}

PBL is a highly aggressive lymphoma strongly associated with immunosuppression, in particular with HIV infection [1]. Nevertheless, PBL has also been described in HIV-negative individuals, usually in association with other causes of immunosuppression (steroid therapy for autoimmune disorders, solid organ transplantation), pre-existing lymphoproliferative diseases or in elderly patients. PBL mainly occurs in men (75\%) with a mean age of 39 years in HIV-positive and 58 years in HIV-negative patients [3]. The disease shows a marked propensity to involve the oral cavity, while the most common extraoral sites are the gastrointestinal tract, lymph nodes and skin. The frequency of oral involvement is higher in HIV-positive (58\%) than in HIV-negative patients (16\%). Other less common localizations include the central nervous system, paranasal sinus, mediastinum, lungs, liver, testes, retroperitoneum, parotid gland and soft tissues. Bone marrow involvement has been reported in 30\% of cases in both HIV-positive and negative individuals. Irrespective of the HIV status, approximately $60 \%$ of patients present with rapidly progressive, destructive, advanced-stage disease; B symptoms tend to be more common in HIV-unrelated cases [3].

The pathogenesis of PBL is currently unclear. The neoplastic cell derives from a post-germinal centre activated B-cell that has already undergone somatic hypermutation and class switching recombination and is in the process of becoming a plasma cell [1]. Mutations and chromosomal aberrations that lead to the development of the malignancy are likely to occur during this transition. Rearrangements of the oncogene $M Y C$ occur in up to $47 \%$ of HIV-related PBL cases and end up with overcoming the regulatory effects of its repressors Bcl-6 and BLIMP-1, thus allowing cells to proliferate without control. Moreover, MYC translocations are thought to contribute to the plasmablastic morphology and to a worse prognosis $[4,5]$. The HIV infection seems to correlate with depth and duration of immunodeficiency and the loss of immune control over oncogenic viral infections - specifically EBV and HHV-8. A chronic antigenic stimulation and the presence of a persistent inflammatory state leads in turn to B-cell dysfunctional proliferation and gives rise to a monoclonal population [1]. The strong association with EBV infection is demonstrated by the expression of EBER, detectable in 80 and $46 \%$ of HIV-positive and negative cases, respectively.

Making a diagnosis of PBL is always challenging: the immunophenotype resembles that of plasma cell neoplasms, as PBL cells typically lack expression of B-cell markers (CD19, CD20, PAX-5) and show little to no expression of leukocyte common antigen CD45, whereas the plasma cell markers CD79a, IRF4/MUM-1, BLIMP-1, CD38 and CD138 are usually highly expressed. The proliferation rate is generally high, with Ki-67 expression higher than 60\% [3]. Association with HIV and/or EBV infection, alongside the absence of monoclonal paraproteinemia, hypercalcemia, renal dysfunction and lytic bone lesions are the key features that favour the diagnosis of PBL instead of MM.

Given its rarity and peculiar features, no standard of care can be established for PBL patients, whose prognosis remains very poor. Early relapses and subsequent chemotherapy resistance characterize the clinical course of the disease. A large literature review of 248 cases of PBL, 50\% of which had received cyclophosphamide, doxorubicin, vincristine, prednisone (CHOP) or CHOP-like regimens, while $23 \%$ had been treated with more intensive regimens, such etoposide, vincristine, doxorubicin, cyclophosphamide, prednisone $(\mathrm{EPOCH})$, hyperfractionated cyclophosphamide, vincristine, doxorubicin, dexamethasone (hyper-CVAD) and cyclophosphamide, vincristine, doxorubicin, methotrexate alternating with ifosfamide, etoposide, cytarabine (CODOX-M/IVAC), showed an overall response rate to chemotherapy of $77 \%$, with $46 \%$ of patients achieving a CR and $31 \%$ a partial response [6]. The median progression-free and overall survival (OS) of PBL patients range between 6 and 7 months and 1113 months respectively, with no differences between 
Table 1 Recent case reports in which antimyeloma drugs have been applied in HIV-negative PBL patients

\begin{tabular}{llllll}
\hline Agent & Author, year & Combination & Patients & Setting & Outcomes \\
\hline Bortezomib & Saba, 2013 [8] & Single agent & 1 & Salvage & PR; DOR 5 months; death \\
& Yan, 2014 [9] & Bor + Rituximab + Dex & 1 & Salvage & nCR; 3+ months post-ASCT \\
& Castillo, 2015 [10] & Bor + DA-EPOCH & $3(*)$ & First-line & 3 CR; DOR 12+, 18+, 24+ months \\
& Hirosawa, 2015 [1] & Single agent & 1 & Salvage & Transient regression \\
& Fedele, 2016 [12] & Bor + DA-EPOCH & 1 & First-line & CR; DOR 24+ months \\
& Cencini, 2016 [13] & Bor + COMP & 1 & First-line & CR; DOR 12 months \\
Lenalidomide & Carras, 2015 [14] & Single agent & 1 & Salvage & CR; DOR 6 months \\
& Schmit, 2017 [15] & Len + cyclo + dex & 1 & First-line & CR; DOR 24+ months \\
\hline
\end{tabular}

(*) 2 HIV-positive patients included

Bor bortezomib, Dex dexamethasone, DA-EPOCH dose-adjusted EPOCH, COMP same as CHOP, but with liposomal doxorubicin, Len lenalidomide, Cyclo cyclophosphamide. $P R$ partial response, $(n) C R$ (near) complete response, DOR duration of response, $A S C T$ autologous transplantation. The sign " + " indicates an ongoing response at the moment of the report

$\mathrm{CHOP} / \mathrm{CHOP}-$ like and more intensive regimens. ASCT may play a role in improving the outcomes of $\mathrm{CR}$ patients [7]. Untreated patients invariably die within a few months (median survival: 3 months). In HIV-infected patients, the use of highly active antiretroviral therapy is recommended, as it seems to be associated with improved survival. However, the strongest prognostic factor for these patients remains the achievement of a CR with chemotherapy, which is associated with a median OS of 48 months compared with 3 months for patients without a CR [6].

On the basis of the plasmacytic differentiation of this lymphoma, given that a plasmablast is an activated B-cell undergoing the process of becoming a plasma cell, PBL is ontogenetically collocated between an aggressive post-germinal centre B-cell non-Hodgkin lymphoma and MM. For this reason, the use of antimyeloma agents - like bortezomib, thalidomide or lenalidomide - may have a sound rationale, although the experience with these agents is rather limited. More specifically, they have been predominantly combined with more standard lymphoma-directed regimens, such as $\mathrm{CHOP}$ or $\mathrm{EPOCH}$ [8-15], rather than applied in MM-like treatment algorithms, also considering at least one ASCT as part of the frontline approach. Case reports accounting for the use of either bortezomib or lenalidomide, applied as single agents or within combinations in both untreated and relapsing PBL patients, have been published in the last few years, sometimes with considerable benefit (Table 1). Our approach was instead based on the full application of a standard MM treatment, with a double ASCT consolidation, and to the best of our knowledge it represents the only reported experience so far. It is important to note, however, that despite the intrinsic aggressiveness of the disease, the early stage at presentation (which accounted for a low International Prognostic Index) may have had a favourable impact on the long-term prognosis of the patient. This treatment was overall well tolerated in an otherwise healthy patient who was considered eligible for a double ASCT, which still represents the standard of care at most Italian institutions for newly diagnosed MM patients who are candidates for an intensive approach. We believe that this approach may represent a possible alternative to intensive lymphoma-directed therapies.

\section{Abbreviations}

ASCT: Autologous stem cell transplantation; CHOP: Cyclophosphamide doxorubicin, vincristine, prednisone; CODOX-M/IVAC: Cyclophosphamide, vincristine, doxorubicin, methotrexate alternating with ifosfamide, etoposide, cytarabine; CR: Complete response; CT: Computed tomography;

EPOCH: Etoposide, vincristine, doxorubicin, cyclophosphamide, prednisone; hyper-CVAD: Hyperfractionated cyclophosphamide, vincristine, doxorubicin, dexamethasone; MM: Multiple myeloma; OS: Overall survival;

PBL: Plasmablastic lymphoma; PBSC: Peripheral blood stem cell; PET: Positron emission tomography

\section{Availability of data and materials}

All data generated or analyzed during this study are included in this published article.

\section{Authors' contributions}

$A B, L N, V S$ and PLZ analyzed and interpreted the patient data regarding the hematological disease and the transplant. MC provided advice for treatment and analysed and interpreted the patient data regarding the hematological disease. CA performed immunoistochemical evaluation. AB, LA and PLZ were major contributor in writing the manuscript. All authors read, revised and approved the final manuscript.

\section{Ethics approval and consent to participate}

This retrospective study was approved by our institutional board and by our Ethical Committee (Comitato Etico Indipendente Policlinico S.Orsola-Malpighi di Bologna) and has been performed in accordance with the ethical standards of the Declaration of Helsinki.

\section{Consent for publication}

The patient provided written informed consent to publish her data.

\section{Competing interests}

The authors declare that they have no competing interests.

\section{Publisher's Note}

Springer Nature remains neutral with regard to jurisdictional claims in published maps and institutional affiliations. 
Received: 18 January 2018 Accepted: 30 May 2018

Published online: 08 June 2018

\section{References}

1. Campo E, Stein H, Harris NL. Plasmablastic lymphoma In: WHO Classification of Tumours of Haematopoietic and Lymphoid Tissues, Swerdlow SH, Campo E, Harris NL, et al, Eds. IARC Press, Lyon, France, Revised 4th edition; 2017. p. 317, 321-322.

2. Cavo M, Zamagni E, Tosi P, Cellini C, Cangini D, Tacchetti $P$, et al. First-line therapy with thalidomide and dexamethasone in preparation for autologous stem cell transplantation for multiple myeloma. Haematologica. 2004;89:826-31.

3. Castillo JJ, Bibas M, Miranda RN. The biology and treatment of plasmablastic lymphoma. Blood. 2015;125:2323-30.

4. Taddesse-Heath L, Meloni-Ehrig A, Scheerle J, Kelly JC, Jaffe ES. Plasmablastic lymphoma with MYC translocation: evidence for a common pathway in the generation of plasmablastic features. Mod Pathol. 2010;23:991-9.

5. Valera A, Balagué O, Colomo L, Martínez A, Delabie J, Taddesse-Heath L, et al. Ig/MYC rearrangements are the main cytogenetic alteration in plasmablastic lymphomas. Am J Surg Pathol. 2010;34:1686-94.

6. Castillo JJ, Winer ES, Stachurski D, Perez K, Jabbour M, Milani C, et al. Prognostic factors in chemotherapy-treated patients with HIV-associated plasmablastic lymphoma. Oncologist. 2010;15:293-9.

7. Al-Malki MM, Castillo JJ, Sloan JM, Re A. Hematopoietic stem cell transplantation for plasmablastic lymphoma: a review. Biol Blood Marrow Transplant. 2014;20:1877-84.

8. Saba NS, Dang D, Saba J, Cao C, Janbain M, Maalouf B, et al. Bortezomib in plasmablastic lymphoma: a case report and review of the literature. Onkologie. 2013;36:287-91.

9. Yan M, dong Z, Zhao F, Chauncey T, Deauna-Limayo D, Wang-Rodriguez J, et al. CD20-positive plasmablastic lymphoma with excellent response to bortezomib combined with rituximab. Eur J Haematol. 2014;93:77-80.

10. Castillo JJ, Reagan JL, Sikov WM, Winer ES. Bortezomib in combination with infusional dose-adjusted EPOCH for the treatment of plasmablastic lymphoma. Br J Haematol. 2015;169:352-5.

11. Hirosawa M, Morimoto H, Shibuya R, Shimajiri S, Tsukada J. A striking response of plasmablastic lymphoma of the oral cavity to bortezomib: a case report. Biomark Res. 2015;3:28.

12. Fedele PL, Gregory GP, Gilbertson M, Shortt J, Kumar B, Opat S, et al. Infusional dose-adjusted EPOCH plus bortezomib for the treatment of plasmablastic lymphoma. Ann Hematol. 2016;95:667-8.

13. Cencini E, Fabbri A, Guerrini S, Mazzei MA, Rossi V, Bocchia M. Long-term remission in a case of plasmablastic lymphoma treated with COMP (cyclophosphamide, liposomal doxorubicin, vincristine, prednisone) and bortezomib. Eur J Haematol. 2016;96:650-4.

14. Carras S, Regny C, Peoc'h M, Gervasoni J, Gressin R, Cahn JY, et al. Dramatic efficacy of low dose lenalidomide as single agent in a patient with refractory gastric non-human immunodeficiency virus-associated plasmablastic lymphoma. Leuk Lymphoma. 2015;56:2986-8.

15. Schmit JM, DeLaune J, Norkin M, Grosbach A. A case of plasmablastic lymphoma achieving complete response and durable remission after lenalidomide-based therapy. Oncol Rest Treat. 2017:40:46-8.

\section{Ready to submit your research? Choose BMC and benefit from:}

- fast, convenient online submission

- thorough peer review by experienced researchers in your field

- rapid publication on acceptance

- support for research data, including large and complex data types

- gold Open Access which fosters wider collaboration and increased citations

- maximum visibility for your research: over $100 \mathrm{M}$ website views per year

At BMC, research is always in progress.

Learn more biomedcentral.com/submissions 